\title{
Orthogonal Polynomial Contrasts and Applications to Age-Period-Cohort Models
}

\author{
Benjamin Elbers* \\ Department of Sociology \\ Columbia University
}

\begin{abstract}
Many regression modeling problems involve ordinal predictors with equally spaced values, such as age or income groups. Orthogonal polynomial contrasts provide a useful way to incorporate the information that the groups are ordered, unlike dummy or effects coding. This technique has been useful to separate the linear and non-linear effects in age-period-cohort (APC) models. This paper addresses three practical issues when implementing orthogonal polynomial contrasts: First, if the linear effect is to be interpreted, it should be identical to the linear effect that we obtain if we treat the predictor as a metric variable. Second, if the data is unbalanced, weighted orthogonal contrasts need to be used to obtain unbiased estimates. Third, by dropping-higher order terms, orthogonal polynomials can easily be used to smooth non-linear estimates. Two examples from the APC literature are discussed, and it is found that the use of unweighted polynomials has led to small biases in the results. A R function is provided that implements weighted polynomials with an interpretable linear term.
\end{abstract}

\section{Introduction}

Since at least Holford (1983), orthogonal polynomial contrasts have been used in the literature on age-period-cohort (APC) models. In the usual setting, we have an outcome $\mathbf{y}$ that is cross-classified by $I$ age groups and $J$ period groups (say, in 5-year intervals), from which we can obtain $K=I+J-1$ birth cohort groups. The goal is then to model the outcome as dependent on age, period, and cohort effects. We assume the basic "APC accounting model," where the outcome $Y_{r}$ for observation $r$ is modeled as

$$
Y_{r}=\beta_{0}+\sum_{i=1}^{I} \alpha_{i} X_{r i}+\sum_{j=1}^{J} \pi_{j} X_{r j}+\sum_{k=1}^{K} \gamma_{k} X_{r k}+\epsilon_{r},
$$

${ }^{*}$ be2239@columbia.edu 
where $\alpha_{i}$ stands for the $i$ th age coefficient, $\pi_{j}$ for the $j$ th period coefficient, and $\gamma_{k}$ for the $k$ th cohort coefficient. The basic problem in estimating such as model is the linear dependency age $=$ period - cohort. Mathematically, the problem can be expressed in terms of the design matrix $\mathbf{X}$. Even if we omit one of each $\alpha_{i}, \pi_{i}$, and $\gamma_{i}$ as the reference category (or use sum-to-zero contrasts), this matrix is still rank-deficient one. This means that we cannot obtain the usual least-squares estimate

$$
\hat{\boldsymbol{\beta}}=\left(\mathbf{X}^{T} \mathbf{X}\right)^{-1} \mathbf{X}^{T} \mathbf{y}
$$

as the inverse of $\mathbf{X}^{T} \mathbf{X}$ is not defined. One additional constraint, such that one of the parameter values is equal to zero (say, $\pi_{3}=0$ ), or that two parameter values are equal to one another (say, $\alpha_{1}=\alpha_{2}$ ), would suffice to make the system of equations identified, and to make $\mathbf{X}^{T} \mathbf{X}$ invertible. While such an additional assumption may seem trivial, it can have severe effects on the parameter estimates (Rodgers, 1982).

Many attempts to circumvent the APC identification problem have been proposed, but these techniques often boil down to imposing an arbitrary constraint (for a discussion, see O'Brien, 2011; Luo et al., 2016; Fosse and Winship, 2018). However, even without imposing a constraint, the data is informative about certain aspects of the possible effects. Holford (1983) first used orthogonal polynomials to separate the linear from the non-linear effects, showing that only the linear terms are not identified. In a series of papers, Fosse and Winship (2018; 2019a; 2019b) have shown how the coefficients can be further restricted through a series of weak assumptions.

This paper makes three contributions, all of which regard the implementation of orthogonal polynomials. First, it is shown that a simple adjustment to the contrast matrix makes it possible to retain an interpretable linear term. Statistical software packages usually provide a linear term that is not interpretable on the scale of the predictor variable, which hinders interpretation. Second, in practice, one often encounters situations where the design matrix is not balanced, i.e. the groups are of unequal sizes. In this case, weighted polynomials contrasts need to be used to obtain unbiased estimates. Third, it is shown that orthogonal polynomials can be used to obtain smoothed estimates of the linear effects.

The paper concludes by reanalzying two examples from the APC literature, Holford (1983) and Fosse and Winship (2019a). While the three contributions concern any use of orthogonal polynomials, APC models provide a good illustration. his will even be necessarily the case, at least for one of the age, period, and cohort effects.

\section{Orthogonal Polynomial Constrasts}

Assume we observe some continuous predictor $\mathbf{y}$ for $n$ observations, such that $\mathbf{y}$ is a $n \times 1$ column vector. We want to use a regression model to investigate how $\mathbf{y}$ depends on an ordinal categorical predictor with $g$ groups, and possibly some additional predictors. To set up the problem, we construct a group membership matrix $\mathbf{G}$ of dimension $n \times g$, where $\mathbf{G}_{i j}=1$ if observation $i$ belongs to group $j$, and 0 otherwise. For concreteness and without 
loss of generality, we assume that we observe data for four age groups, 0-9, 10-19, 20-29, and 30-39, and that there are no other predictors. If we observe two data points for each group, then $\mathbf{G}$ would have the following form:

$$
\mathbf{G}=\left[\begin{array}{llll}
1 & 0 & 0 & 0 \\
1 & 0 & 0 & 0 \\
0 & 1 & 0 & 0 \\
0 & 1 & 0 & 0 \\
0 & 0 & 1 & 0 \\
0 & 0 & 1 & 0 \\
0 & 0 & 0 & 1 \\
0 & 0 & 0 & 1
\end{array}\right]
$$

There are now several possible modeling strategies. A common choice for modeling this problem is to use dummy-coding with coding matrix

$$
\mathbf{C}_{\mathrm{d}}=\left[\begin{array}{llll}
1 & 0 & 0 & 0 \\
1 & 1 & 0 & 0 \\
1 & 0 & 1 & 0 \\
1 & 0 & 0 & 1
\end{array}\right]
$$

where the first group is omitted as the reference category. We can obtain the design matrix by $\mathbf{X}_{\mathrm{d}}=\mathbf{G C}_{\mathrm{d}}$, and then solve the usual least-squares equation as

$$
\hat{\boldsymbol{\beta}}_{\mathrm{d}}=\left(\mathbf{X}_{\mathrm{d}}^{T} \mathbf{X}_{\mathrm{d}}\right)^{-1} \mathbf{X}_{\mathrm{d}}^{T} \mathbf{y}
$$

The model can also be written as

$$
Y_{i}=\beta_{\mathrm{d}, 0}+\beta_{\mathrm{d}, 1} x_{i 1}+\beta_{\mathrm{d}, 2} x_{i 2}+\beta_{\mathrm{d}, 3} x_{i 3}+\epsilon_{i}
$$

where $x_{i 1}=1$ if observation $i$ belongs to age group 10-19, and 0 otherwise, $x_{i 2}=1$ if observation $i$ belongs to age group 20-29, and 0 otherwise, and $x_{i 3}=1$ if observation $i$ belongs to age group 30-39, and 0 otherwise. This is a standard model, and one where the coefficients $\hat{\boldsymbol{\beta}}_{d}$ are easy to interpret. If we let $\bar{y}_{j}$ denote the mean of the outcome for the $j$ th age group, then $\hat{\beta}_{d, 0}=\bar{y}_{1}, \hat{\beta}_{d, 1}=\bar{y}_{2}-\bar{y}_{1}$, etc.

For another possible model we construct a vector $\mathbf{k}$, which for each observation records the midpoint of the age category it belongs to. For instance, if we have two observations for each age category, then $\mathbf{k}=(5,5,15,15,25,25,35,35)$. We can then construct another model as follows:

$$
Y_{i}=\beta_{\mathrm{p}, 0}+\beta_{\mathrm{p}, 1} k_{i}+\beta_{\mathrm{p}, 2} k_{i}^{2}+\beta_{\mathrm{p}, 3} k_{i}^{3}+\epsilon_{i}
$$

The coding matrix for this model is

$$
\mathbf{C}_{p}=\left[\begin{array}{cccc}
1 & 5 & 25 & 125 \\
1 & 15 & 225 & 3375 \\
1 & 25 & 625 & 15625 \\
1 & 35 & 1225 & 42875
\end{array}\right]
$$


thus, we can obtain the least-squares coefficients $\hat{\boldsymbol{\beta}}_{p}$ through the design matrix $\mathbf{X}_{\mathbf{p}}=\mathbf{G C}_{\mathrm{p}}$. The coefficients $\hat{\boldsymbol{\beta}}_{p}$ are now not as easy to interpret, but the model is still of interest. This model treats the age groups as a metric variable, effectively incorporating the information that the predictor is an ordinal variable. However, as the higher-order terms are directly derived from $\mathbf{k}$, the predictors will be highly correlated.

In practice, therefore, orthogonal polynomials are used, leading to a third model of the form (notation adapted from Emerson, 1968):

$$
Y_{i}=\beta_{0}^{*} p_{0}\left(k_{i}\right)+\beta_{1}^{*} p_{1}\left(k_{i}\right)+\beta_{2}^{*} p_{2}\left(k_{i}\right)+\beta_{3}^{*} p_{3}\left(k_{i}\right)+\epsilon_{i}
$$

where $p_{j}(k)$ is a polynomial of degree $j$ in $k$ and where the $p_{j}$ are orthogonal functions in the sense that

$$
\sum_{i=1}^{n} p_{j}\left(k_{i}\right) p_{l}\left(k_{i}\right)=0 \quad \text { if } j \neq l
$$

Another way to put this is that all columns of the design matrix will be mutually orthogonal. For the balanced case, where all groups are of the same size, one can use tables for orthogonal coefficients to find $p_{j}\left(k_{i}\right)$. For instance, Draper and Smith (1998, p. 466) provide the following coding table for $g=4$ :

$$
\mathbf{C}^{*}=\left[\begin{array}{cccc}
1 & -3 & 1 & -1 \\
1 & -1 & -1 & 3 \\
1 & 1 & -1 & -3 \\
1 & 3 & 1 & 1
\end{array}\right]
$$

Model (4) has the advantage that the coefficient $\beta_{1}$ captures only the linear association between the age groups and the outcome. This makes it possible to test whether the higher-order polynomial terms are significant, and whether they could possibly be discarded to obtain a more parsimonious model.

It is important to recognize that the models (2), (3), (4) are equivalent in the sense that their design matrices span the same column space. Hence, the predictions from these models will be identical, as will be all model fit statistics. Which model we prefer comes down to deciding which set of coefficients $\hat{\boldsymbol{\beta}}_{\mathrm{d}}, \hat{\boldsymbol{\beta}}_{p}$, or $\hat{\boldsymbol{\beta}}^{*}$ we prefer.

\section{Interpreting the linear effect}

One problem in interpreting the coefficients $\hat{\boldsymbol{\beta}}^{*}$ of the orthogonal polynomial model is that the coefficients are arbitrary. The matrix $\mathbf{C}^{*}$ is arbitrary in the sense that any column can be scaled upwards or downwards by a constant non-zero factor, without changing the fact that all columns in the design matrix are mutually orthogonal. For instance, statistical software packages (contr . poly in $\mathrm{R}$ is used here) may provide the following matrix for the 
4-group case:

$$
\mathbf{C}^{* *}=\left[\begin{array}{cccc}
1 & -3 / \sqrt{20} & 1 / 2 & -1 / \sqrt{20} \\
1 & -1 / \sqrt{20} & -1 / 2 & 3 / \sqrt{20} \\
1 & 1 / \sqrt{20} & -1 / 2 & -3 / \sqrt{20} \\
1 & 3 / \sqrt{20} & 1 / 2 & 1 / \sqrt{20}
\end{array}\right] .
$$

In this matrix, all columns except for the intercept column are of unit length. Necessarily, we obtain different sets of coefficients depending on whether we use $\mathbf{C}^{*}, \mathbf{C}^{* *}$, or any of the other infinite possibilities.

This is unproblematic, as it is not desirable to directly interpret the size of the higherorder coefficients anyway. However, it is often useful to be able to retain the interpretability of the linear coefficient. Intuitively, the linear effect should reflect the change in $y$ that is produced by a one-unit change in the predictor, for some useful definition of "unit." Although we have age groups here, it would thus be useful to report the linear coefficient for a one-year change in age. This is the same coefficient that would be obtained if we simply regressed the outcome on the midpoints $\mathbf{k}$, i.e. the reduced model

$$
Y_{i}=\beta_{\mathrm{r}, 0}+\beta_{\mathrm{r}, 1} k_{i}+\epsilon_{i} .
$$

The coding matrix that achieves this result is simple to obtain. Collect the midpoints for each group in a vector $\mathbf{k}^{*}=(5,15,25,35)$, and subtract the mean of this vector, $\bar{k}^{*}=20$. The resulting vector will have the appropriate step size that is required by the width of the categories. For instance, if age is grouped in 10-year categories, all first differences of the vector $\mathbf{k}^{*}-\bar{k}^{*}$ will be 10 . For this example, we then obtain the desired contrast matrix

$$
\mathbf{C}=\left[\begin{array}{cccc}
1 & -15 & s & -t \\
1 & -5 & -s & 3 t \\
1 & 5 & -s & -3 t \\
1 & 15 & s & t
\end{array}\right]
$$

where $s \neq 0$ and $t \neq 0$ are arbitrary constants. The coefficient $\beta_{1}$ obtained from this model will be identical to the coefficient $\beta_{\mathrm{r}, 1}$ in model (5). It is easily interpretable as the change in $y$ that is associated with a one-year increase in age.

\section{Accounting for unbalanced data}

Orthogonal polynomial contrasts are often applied in experimental settings where the number of observations in each group is the same. The same assumption is often made in textbooks (e.g., Draper and Smith, 1998). It is thus worth repeating here that when we apply any of the coding matrices $\mathbf{C}^{*}, \mathbf{C}^{* *}$ or $\mathbf{C}$ to a group membership matrix $\mathbf{G}$ where the number of observations is not equal in each group, the design matrix $\mathbf{X}$ will not have columns that are mutually orthogonal. This defeats the purpose of using orthogonal polynomials, and is not the desired result. 
We can illustrate this problem through a simple example. Assume that we drop from the matrix $\mathbf{G}$ above one observation from each of the last two age groups. We then obtain the matrix $\mathbf{G}_{r}$ of dimension $6 \times 4$. In this matrix, the first two age groups have two observations each, while the last two age groups are now represented by only one observation each. We then apply the unweighted contrast matrix $\mathbf{C}$ with $s=1, t=1$ to obtain

$$
\mathbf{X}=\mathbf{G}_{r} \mathbf{C}=\left[\begin{array}{cccc}
1 & -15 & 1 & -1 \\
1 & -15 & 1 & -1 \\
1 & -5 & -1 & 3 \\
1 & -5 & -1 & 3 \\
1 & 5 & -1 & -3 \\
1 & 15 & 1 & 1
\end{array}\right]
$$

While the contrast matrix $\mathbf{C}$ has columns that are pairwise orthogonal, this is no longer true for the matrix $\mathbf{X}$ :

$$
\mathbf{X}^{T} \mathbf{X}=\left[\begin{array}{cccc}
6 & -20 & 0 & 2 \\
-20 & 750 & -10 & 0 \\
0 & -10 & 6 & -4 \\
2 & 0 & -4 & 30
\end{array}\right]
$$

The correct contrast matrix needs to be obtained through the use of formulas for weighted polynomials, given for instance by Gaito (1965) or Emerson (1968). Again, the norm of the higher-order polynomials is not important, but we can retain the interpretability of the linear term, incorporating the suggestion from the previous section. This term is formed as above using the vector of midpoints $\mathbf{k}^{*}=(5,15,25,35)$, except that we now need to subtract the weighted mean $\bar{k}_{w} \approx 16.67$, obtained using the group weights $(1 / 3,1 / 3,1 / 6,1 / 6)$. For this example, the correct matrix is (approximately, as entries are rounded)

$$
\mathbf{C}_{r}=\left[\begin{array}{cccc}
1 & -11.67 & 0.4087 s & -0.1414 t \\
1 & -1.67 & -0.5059 s & 0.4243 t \\
1 & 8.33 & -0.4328 s & -0.8485 t \\
1 & 18.33 & 0.6251 s & 0.2828 t
\end{array}\right]
$$

where the quadratic and cubic terms were obtained using the R function contr . poly . weighted described in the appendix. The matrix $\mathbf{C}_{r}$ does not have pairwise orthogonal columns when weighted orthogonal polynomials are used.

\section{Smoothing}

To reiterate, choosing orthogonal polynomial contrasts (or any other contrast coding scheme) to model the relationship between the predictor variable and the outcome will not alter the model. The two models will have identical fit statistics and the same predicted values. If we have $d$ groups, both models require fitting $d$ parameters, including the intercept. In the treatment-coded model, the coefficients $\hat{\boldsymbol{\beta}}$ are interpretable as the means of the outcome 

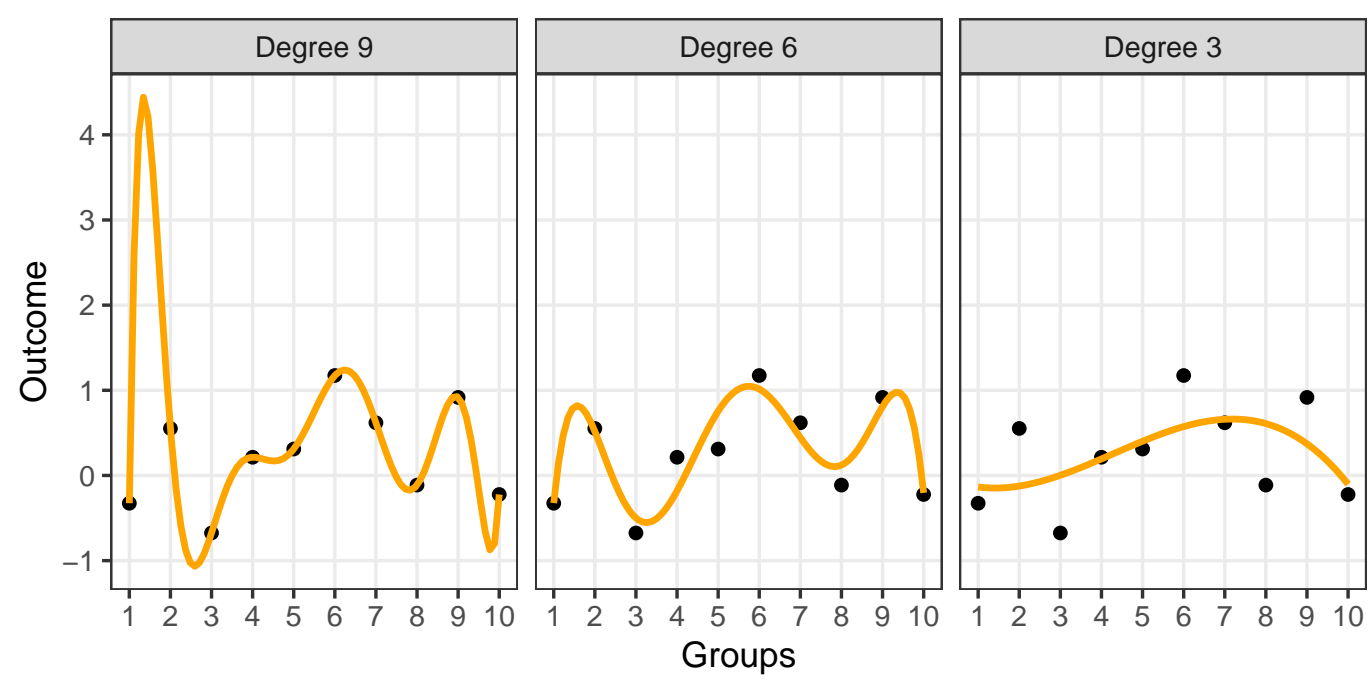

Figure 1: Polynomial fits of different degree to 10 data points drawn from $\operatorname{Normal}(0,1)$

values for the different groups ( $\bar{y}_{j}$ in the above notation), while in the orthogonal polynomial model, the coefficients are associated with the orthogonal polynomial terms. The polynomial function of degree $d$ that is obtained from the orthogonal fit will pass through all $d$ group means $\bar{y}_{j}$, as shown in the left-hand panel of Figure 1. As seen, this function will usually be highly overfit, and often behave bizzarely between two adjacent groups.

To achieve a more stable fit, one can simply drop higher-order polynomial terms. Figure 1 also shows polynomials of degree 6 and 3, both of which provide a smoother fit. (In this case, at most a linear function should be fit, as the data was generated in a way such that there is no relationship between the groups and the outcome.) Formally, deciding how many terms to drop could be done through F-tests. In many cases, a quadratic or cubic polynomial will be enough to capture the relevant trends in the data. Because this approach can "save" a larger number of degrees of freedom, this approach can be of interest in settings where additional covariates enter into the model.

In the APC literature, the smoothing is also of practical relevance for specifying monotonicity constraints. We will return to this point below.

\section{Examples from the APC literature}

\section{A reanalysis of Holford (1983)}

Holford (1983) pioneered the use of orthogonal polynomials for age-period-cohort analysis. For illustration, Holford presented a design matrix for equally spaced age and period intervals $(I=J=3)$. The data can be represented as a $3 \times 3$ contingency table, where each cell $y_{i j}$ contains the outcome of interest. As in a Lexis diagram, the period increases from 
left to right, and age increases from bottom to top:

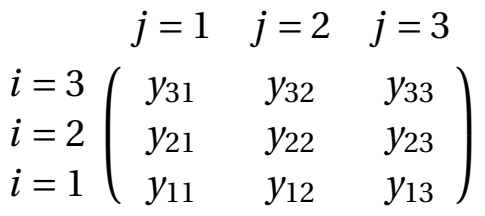

For instance, $y_{12}$ is the outcome for the first age group and second period group. The cohorts can be read off from the diagonals. Starting in the upper left-hand corner, we observe for the first cohort, $k=1$, only one data point, $y_{31}$. The second cohort, $k=2$, consists of two observations $\left(y_{21}, y_{32}\right)$, the third cohort of three observations $\left(y_{11}, y_{22}, y_{33}\right)$, etc. There are therefore $I+J-1=5$ cohorts, but with an unequal number of observations per cohort group. It follows that even if we have an equal number of observations for age and period groups, there will necessarily be an unequal number of observations for cohort groups. Hence, the use of weighted orthogonal polynomials cannot be avoided when dealing with APC models.

Table 1 (also Table 1 in Holford, 1983) shows the corrected design matrix, which accounts for the use of weighted orthogonal polynomials for the cohort coefficients. The coefficients that were corrected are bolded. The columns $A_{L}, P_{L}$, and $C_{L}$ show the linear coefficients for the age, period, and cohort effects, respectively. The columns $A_{\text {quad }}$ and $P_{\text {quad }}$ contain the non-linear coefficients for age and period, respectively. It is easy to verify that the two sets of columns are orthogonal, i.e. $\left\langle A_{L}, A_{\text {quad }}\right\rangle=0$ and $\left\langle P_{L}, P_{\text {quad }}\right\rangle=0$ (where $\langle\cdot\rangle$ denotes the inner product). As we have five cohort groups, we require polynomials up to the fourth degree. It can be verified that the columns $C_{L}, C_{\text {quad }}, C_{\text {cubic }}$, and $C_{\text {quartic }}$ are all pairwise orthogonal. This is not the case in Holford's original design matrix, and that design matrix is thus not able to correctly separate the linear from the non-linear effects.

Using this design matrix, it is possible to estimate the "linearized" APC model as follows:

$$
Y_{r}=\beta_{0}+\underbrace{\alpha_{L} A_{L}+\pi_{L} P_{L}+\gamma_{L} C_{L}}_{\text {linear terms }}+\underbrace{\sum_{i=2}^{I-1} \tilde{\alpha}_{i} A_{r i}+\sum_{j=2}^{J-1} \tilde{\pi}_{j} P_{r j}+\sum_{k=2}^{K-1} \tilde{\gamma}_{k} C_{r k}}_{\text {non-linear terms }}+\epsilon_{r},
$$

where $A_{r i}$ denotes the entry of the age-specific columns of the design matrix for the $r$ th observation and the $i$ th non-linearity (and $P_{r j}, C_{r k}$ equivalently). Note that the design matrix for this model is still rank-deficient one, as the linear dependency $A_{L}=P_{L}-C_{L}$ remains. However, as the linear effects are orthogonal to the non-linear effects, this model can uniquely identify the non-linear effects. The linearized APC model (6) is simply another representation of the "accounting model" in equation (1). Both models will have identical predicted values and identical model fit statistics.

Holford's empirical example concerns rates of prostate cancer deaths for nonwhites in the United States (Holford, 1983, Table 2). The data is cross-classified by seven period groups (1935 to 1965) and seven age groups (50 to 80), each classified in five-year intervals. Coefficient estimates are obtained by a Poisson regression, using the natural logarithm of the mid-period population size in each cell as the offset. To obtain coefficient estimates for 


\begin{tabular}{rrrrrrrrrrr}
\hline$i$ & $j$ & $k$ & $A_{\text {quad }}$ & $P_{\text {quad }}$ & $C_{\text {quad }}$ & $C_{\text {cubic }}$ & $C_{\text {quartic }}$ & $A_{L}$ & $P_{L}$ & $C_{L}$ \\
\hline 1 & 1 & 3 & 1 & 1 & $-\mathbf{4}$ & $\mathbf{0}$ & $\mathbf{2}$ & -1 & -1 & 0 \\
& 2 & 4 & 1 & -2 & $-\mathbf{1}$ & $-\mathbf{1}$ & $\mathbf{- 2}$ & -1 & 0 & 1 \\
& 3 & 5 & 1 & 1 & $\mathbf{8}$ & $\mathbf{1}$ & $\mathbf{1}$ & -1 & 1 & 2 \\
2 & 1 & 2 & -2 & 1 & $-\mathbf{1}$ & $\mathbf{1}$ & $\mathbf{- 2}$ & 0 & -1 & -1 \\
& 2 & 3 & -2 & -2 & $-\mathbf{4}$ & $\mathbf{0}$ & $\mathbf{2}$ & 0 & 0 & 0 \\
& 3 & 4 & -2 & 1 & $-\mathbf{1}$ & $-\mathbf{1}$ & $\mathbf{- 2}$ & 0 & 1 & 1 \\
3 & 1 & 1 & 1 & 1 & $\mathbf{8}$ & $-\mathbf{1}$ & $\mathbf{1}$ & 1 & -1 & -2 \\
& 2 & 2 & 1 & -2 & $-\mathbf{1}$ & $\mathbf{1}$ & $\mathbf{- 2}$ & 1 & 0 & -1 \\
& 3 & 3 & 1 & 1 & $-\mathbf{4}$ & $\mathbf{0}$ & $\mathbf{2}$ & 1 & 1 & 0 \\
\hline
\end{tabular}

Table 1: Corrected design matrix for $I=J=3$

(Bolded entries are changed from Holford, 1983, Table 2)

the non-linear terms, we can construct a design matrix that omits one of the linear terms $A_{L}, P_{L}$, or $C_{L}$. In this example, every age and period group occurs in the design matrix an equal number of times, and weighted orthogonal polynomials are thus only required for the cohort coefficients.

Focusing first on the non-linear effects, Figure 2 shows a comparison between the nonlinear cohort effects obtained using unweighted (Holford, 1983, Table 4) and weighted (this paper) orthogonal polynomials. The results shows that Holford's coefficient estimates are biased upward.

The linear effects cannot be uniquely identified unless additional assumptions are made. For the purpose of illustration, we assume now that the period linear effect is zero, i.e. $\pi_{L}=0$. We can then obtain estimates for $\alpha_{L}$ and $\gamma_{L}$. Under this assumption, Holford's estimates are

$$
\hat{\alpha}_{L}^{*}=0.584 \text { and } \hat{\gamma}_{L}^{*}=0.115 \text {. }
$$

Using weighted orthogonal polynomials and by choosing the linear term to be interpretable on the year scale, we obtain

$$
\hat{\alpha}_{L}=0.117 \text { and } \hat{\gamma}_{L}=0.025 \text {. }
$$

Note that $\hat{\alpha}_{L}^{*}$ is unbiased, and, as we have five-year age groups, the estimates have a simple relationship: $\hat{\alpha}_{L}^{*}=5 \times \hat{\alpha}_{L}$. In principle, this is also true for the cohort linear effect, however, Holford's estimate $\hat{\gamma}_{L}^{*}$ is slightly biased due to the use of unweighted orthogonal polynomials. The difference in interpretation is then simply that Holford's estimates are interpretable as the change in the log rate of cancer incidence that is associated with an increase in one age group, while the estimates $\hat{\alpha}_{L}$ and $\hat{\gamma}_{L}$ are interpretable as the change associated with an increase in one age year. Thus, when disregarding the non-linear terms, and under the assumption that $\pi_{L}=0$, a one-year increase in age is associated with an increase in the log rate of cancer incidence of 0.117 , and a one-year increase in the birth cohort is associated with an increase of 0.025 .

The use of orthogonal polynomials make further improvements to the model possible. Currently, the model contains one intercept, two coefficients for the linear parame- 


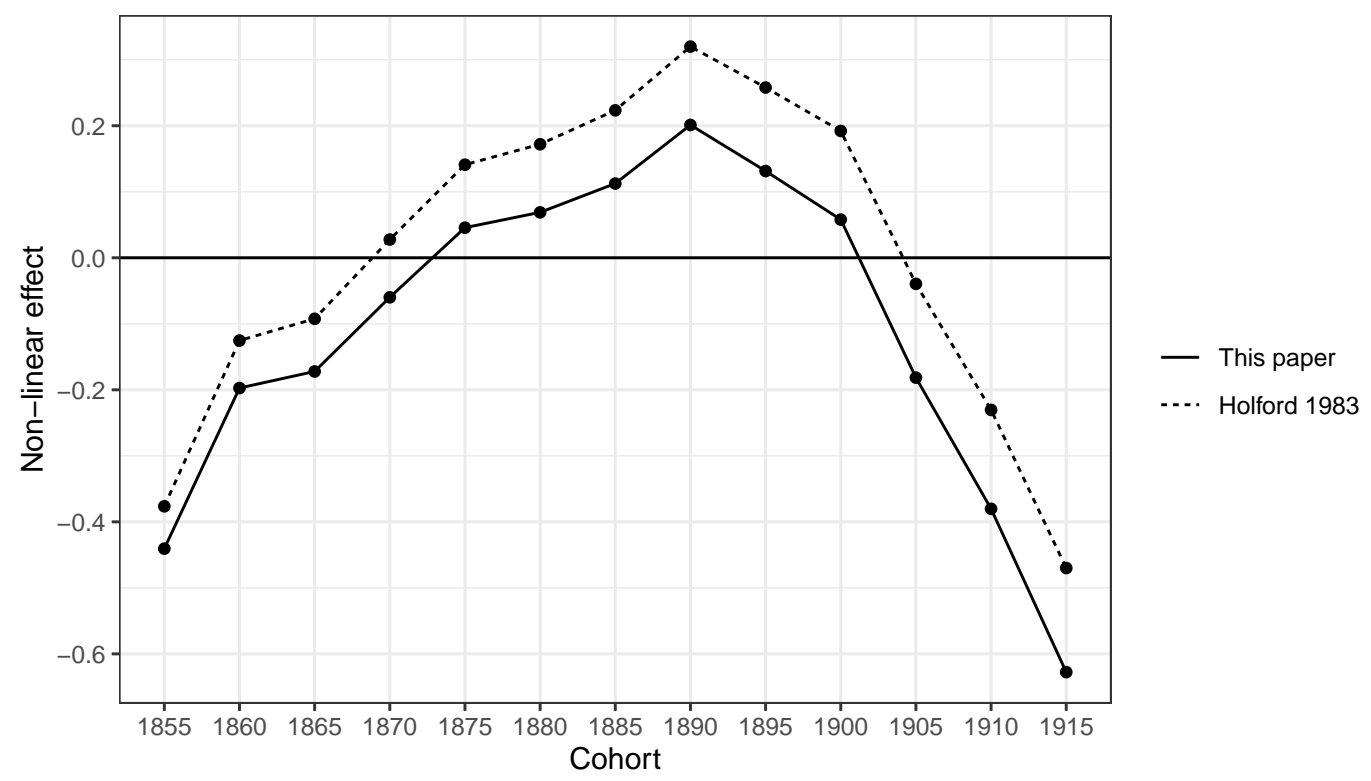

Figure 2: Comparison between cohort non-linearities

ters, and 21 parameters for the non-linear coefficients (5 each for age and period, 11 for cohort), for a total of 24 parameters. To arrive at a more parsimonious model, one can discard higher-order polynomial terms. For the current model, a parsimonious subset of the full model with only 6 parameters-intercept, two parameters for the linear terms, and quadratic terms for age, period, and cohort each-provides an almost identical fit. This does not change the fact that the true linear effects are unidentified, but it avoids overfitting of the non-linear terms.

\section{A reanalysis of Fosse and Winship (2019a)}

Fosse and Winship (2019a) present a unified framework for APC analysis that is based on the linearized APC model. They define the estimable quantities $\theta_{1}=\alpha_{L}+\pi_{L}$ and $\theta_{2}=$ $\gamma_{L}+\pi_{L}$, which can be obtained from the linearized APC model by setting $\pi_{L}=0$. The key insight that Fosse and Winship (2019a) present is that, due to the linear relationship between $\theta_{1}$ and $\theta_{2}$, the unknown values of the linear effects $\alpha_{L}, \pi_{L}$, and $\gamma_{L}$ are constrained to lie on a line in two-dimensional space. This vastly reduces the number of possibilities that these values can take. Next, one can proceed to further reduce the space of possibilities through weak and theoretically motivated assumptions, such as monotonicity constraints (e.g., one can specify that the total age effect should be monotonically increasing), or through the use lower and upper bounds on the linear effects. While not yielding point identification of the linear effects, these techniques can come close.

The empirical example presented in Fosse and Winship (2019a) is based on General Social Survey (GSS) data for $N=23,825$ respondents. The outcome is the number of words correct on a vocabulary quiz of ten items (min: 0 , max: 10, sd: 2.14). Age and period are 


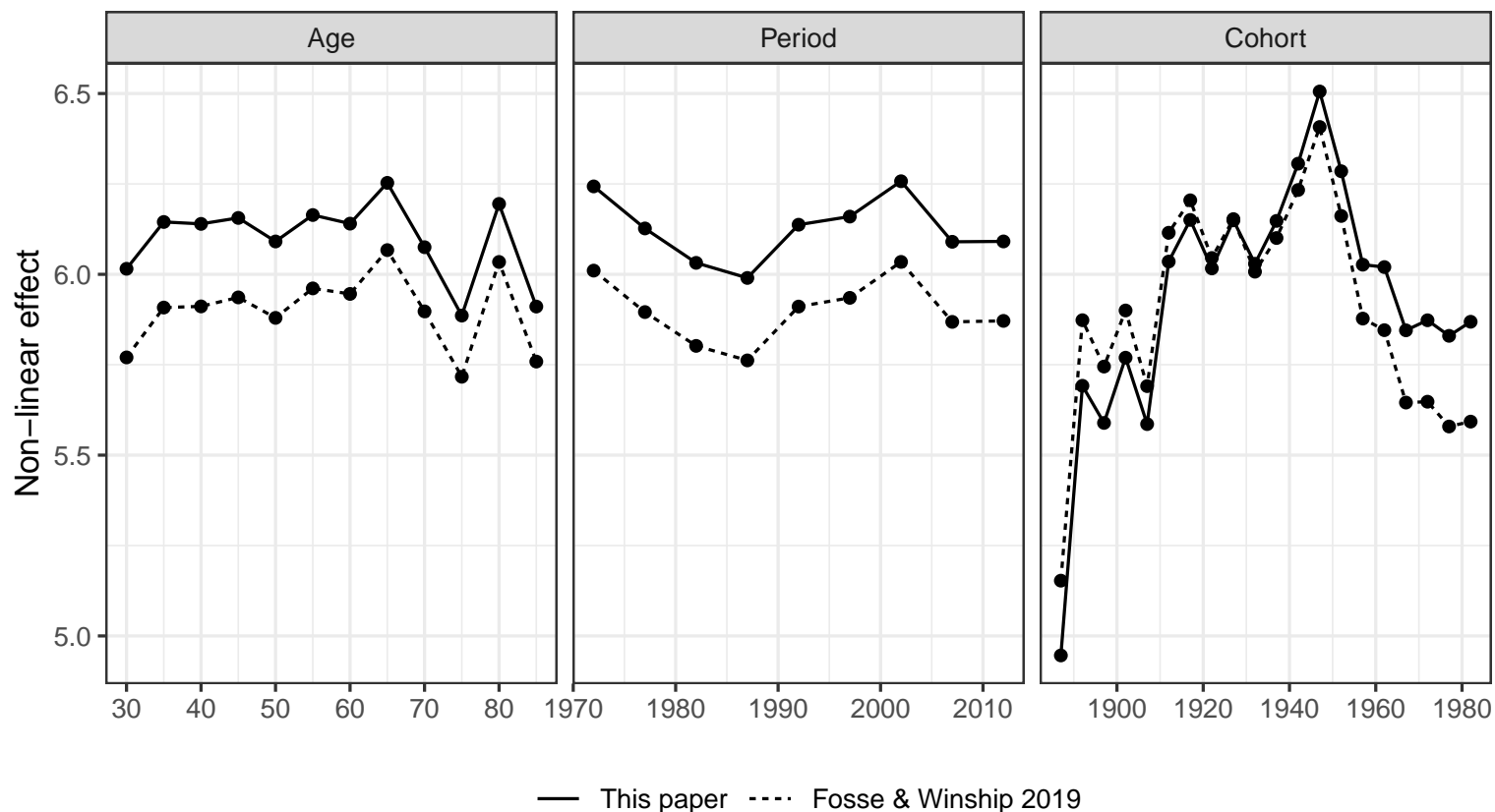

Figure 3: Comparison between non-linearities (including the intercept term)

grouped into five-year intervals. All models are OLS regression models, assuming that the outcome can be modeled as continuous.

In Holford's example above, the unit of analysis was a cell in the contingency table. For the GSS data, the unit of analysis is the individual. This means that, in all likelihood, age, period, and cohort groups will be of unequal sizes. This makes it necessary to apply weighted orthogonal polynomials to all three predictor sets, and we expect that the parameter estimates shown in Fosse and Winship (2019a) are biased for age, period, and cohort effects.

Figure 3 shows the non-linearities for age, period, and cohort effects. To ease comparison with the original figure (Fosse and Winship, 2019a, Suppl. Material, Figure 10), the intercept term has been included. Figure 3 shows that both age and period effects are underestimated in the original paper. The cohort effect shows a smaller bias: for older cohorts, the non-linear effects have been slightly overestimated, while for younger cohorts the effects have been slightly underestimated.

As estimates of $\theta_{1}$ and $\theta_{2}$, Fosse and Winship (2019a) obtain

$$
\hat{\theta}_{1}^{*}=\alpha_{L}+\pi_{L}=-0.122 \text { and } \hat{\theta}_{2}^{*}=\gamma_{L}+\pi_{L}=0.384 .
$$

These two terms are biased because of the use of unweighted polynomials. Disregarding this for the moment, we turn to the question of interpretation. The authors chose a stepsize of 0.1 for the linear term; thus, in order to obtain a linear term that is interpretable as the change in the outcome for one year, we can calculate $-0.122 \times 0.1 / 5=-0.00244$ and $0.384 \times 0.1 / 5=0.00768$. If we assume again that the true linear period effect is zero, these 


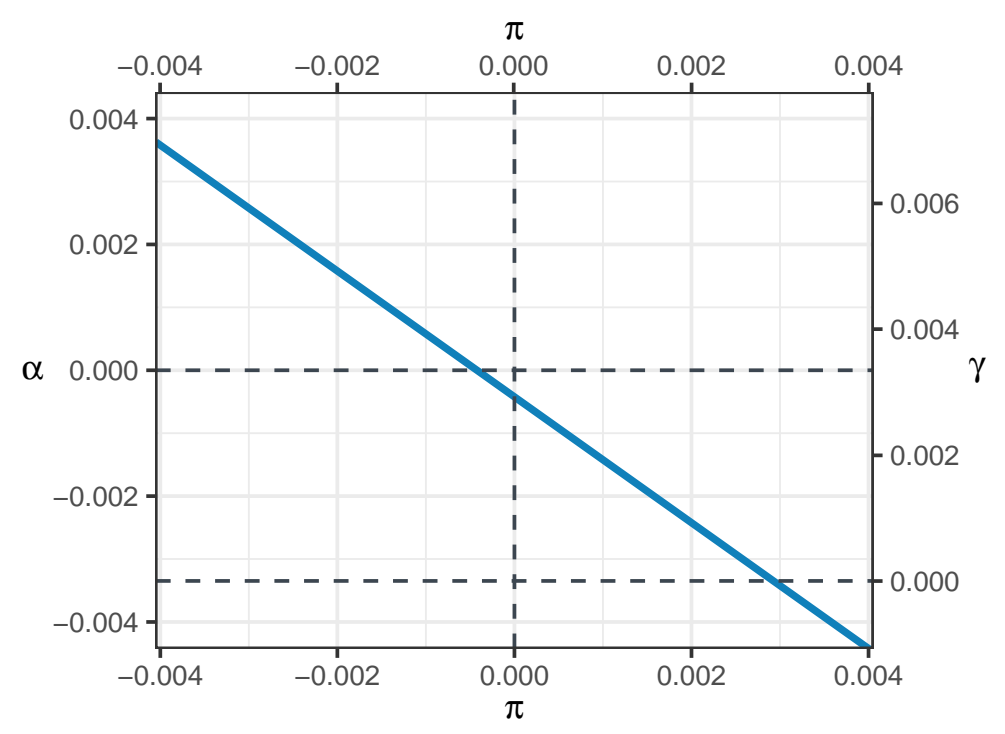

Figure 4: Canonical Solution Line for Corrected Model

two estimates would correspond to the age and cohort linear effect, respectively. Clearly, these effects are estimated to be very small. For instance, the total linear age effect possible in the sample (from age 30 to age 90 ) is predicted to be $-0.00244 \times(90-30) \approx-0.15$, which corresponds to about $7 \%$ of one standard deviation of the outcome.

The unbiased estimated obtained through the use of weighted polynomials are

$$
\hat{\theta}_{1}=\alpha_{L}+\pi_{L}=-0.0004 \text { and } \hat{\theta}_{2}=\gamma_{L}+\pi_{L}=0.0029
$$

We use these estimates to show the canonical solution line in two-dimensional space (Figure 4). Dashed lines are drawn where $\alpha_{L}=0, \pi_{L}=0$, and $\gamma_{L}=0$. The graph shows, for instance, that if we assume that the age linear effect is positive, the period linear effect must be negative, and the cohort linear effect must be greater than $\theta_{2}-\theta_{1}=0.0033$. The difference between Figure 4 and the equivalent figure in Fosse and Winship (2019a, Figure 7a) is that the linear terms are calculated based on the unit of one year. This can make it easier to understand the effects of constraints, such as monotonicity or bounding constraints.

As an example, we consider a monotonicity constraint on the total cohort effect. Due to educational expansion, it can be argued that that the total cohort effect (i.e., linear and non-linear effects) should be monotonically increasing. To achieve this, we have to assume the cohort linear effect to be large enough to offset any declines in the cohort nonlinear effect. There is one problem here: The non-linearities in Figure 3 are likely overfit (we fit one parameter per cohort group), which makes the estimates quite noisy. It would thus be desirable to smooth these effects. A simple way to achieve this is by simply dropping higher-order polynomials. ${ }^{1}$ For this example, we drop polynomial terms beyond the fifth degree.

\footnotetext{
${ }^{1}$ Fosse and Winship (2019a, fn 16) suggest to smooth the Lexis table, which is another way to obtain smoothed estimates.
} 


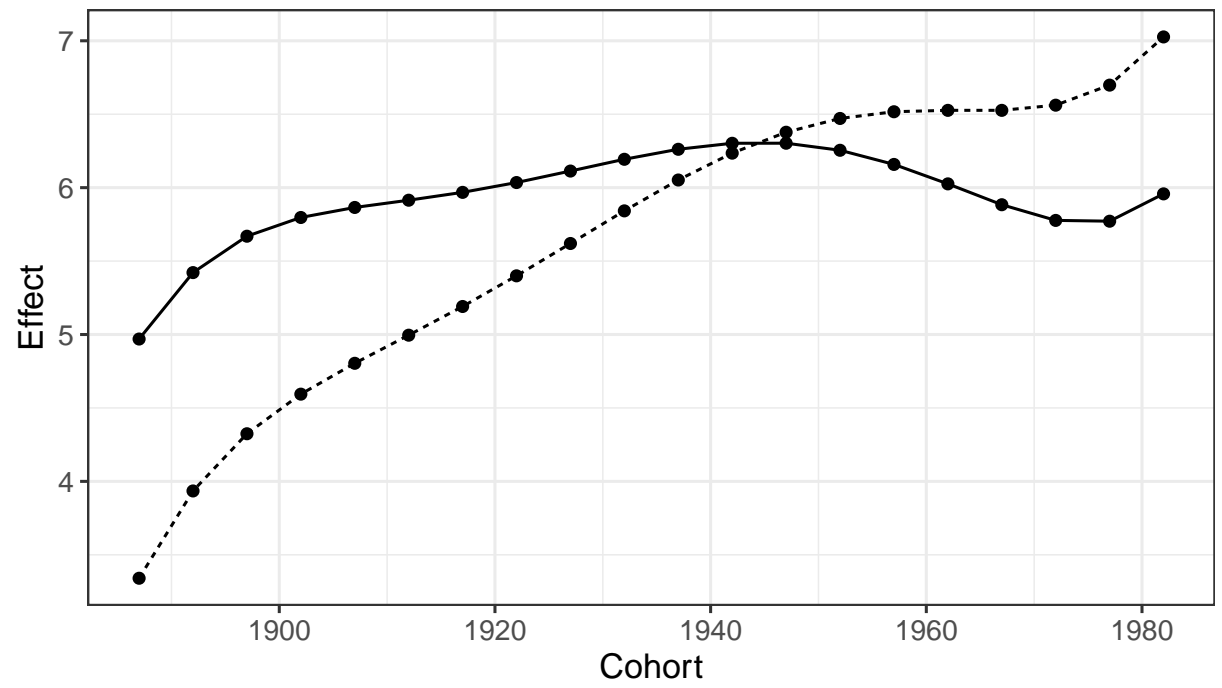

— Non-linear only, poly. of degree 5 -... $\quad$ Under monotonicity constraint

Figure 5: Total smoothed cohort effect (dashed) under monotonicity constraint

Using the smoothed estimates, the largest decline in the non-linear cohort effect occurs between 1962 and 1967, where the difference is -0.142 . The minimum cohort linear effect required to offset any declines would thus be $0.142 / 5=0.028 .^{2}$ The constraint translates into the assumption that a one-year increase in the birth cohort is associated with an increase in vocabulary size of 0.028 , about $1 \%$ of a standard deviation of the outcome. The effect of this constraint on the total cohort effect is shown in Figure 5. Of course, the scenario shown in the figure just represents the minimum, where $\gamma_{L}$ equals 0.028 exactly; higher values for the cohort linear effect are possible under this constraint and will lead lead to steeper cohort total effects.

Because of the interdependencies between the linear parameters, assuming a constraint for the cohort linear effect also impacts the age and period linear effects. We solve

$$
\begin{aligned}
0.0029-\pi_{L} & =\gamma_{L}>0.028 \\
0.0029-\pi_{L} & >0.028 \\
\pi_{L} & <-0.0251
\end{aligned}
$$

and

$$
\begin{aligned}
-0.0004-\alpha_{L} & =\pi_{L}<-0.0251 \\
-0.0004-\alpha_{L} & <-0.0251 \\
\alpha_{L} & >0.0247
\end{aligned}
$$

\footnotetext{
${ }^{2}$ Using the unsmoothed estimates, the minimum cohort linear effect would be 0.052 , almost double the smoothed estimate.
} 
to obtain bounds on the age and period linear effects. Again, these effects are interpretable as the change in the outcome that is associated with a one-year increase in age and period, respectively.

\section{Conclusion}

Orthogonal polynomials can be useful for many applications, but especially so for ageperiod-cohort analysis. By separating the linear from the non-linear effects, the linearized APC allows the point-identification of many parameters and estimable quantities, with the exception of the three linear effects. Through relatively weak assumptions, these effects can be further constrained.

This paper has addressed three issues:

1. As the reanalysis of the examples has shown, it is desirable to obtain interpretable linear effects. This requires just a simple change to the coding matrix.

2. To avoid biased estimates, it is necessary to make use of weighted orthogonal polynomials when the age, period, or cohort groups are unbalanced.

3. Orthogonal polynomials make it easy to smooth the age, period, and cohort effects. When implementing monotonicity constraints, it is highly desirable to use smoothed estimates in order to avoid that unreasonably large increases and decreases affect the results.

\section{Appendix: R package}

The default procedures in $\mathrm{R}$ and Stata do not provide an interpretable linear term, and in $\mathrm{R}$, there is no built-in functionality to obtain weighted orthogonal polynomial contrasts. In Stata, weighted orthogonal contrasts can be obtained using the command contrast pw. <var>.

To gain equivalent functionality in $\mathrm{R}$, and to obtain the interpretable linear term directly, the R package weightedcontrasts is provided. The function contr . poly . weighted ( $f$, weights $=$ NULL, width $=1$ ) takes in a vector of data $f$ and an argument width, which is the size of the groups. For instance, for age groups 0-9, 10-19, etc., width should be set to 10 . The weights are usually obtained from the vector $f$, but can also be specified using the optional weights argument. The function will then create a contrast matrix that can be used in regression models, just as the built-in contrast functions.

\section{References}

Draper, Norman R. and Harry Smith. 1998. Applied Regression Analysis. Third edition. 
Emerson, Phillip L. 1968. "Numerical Construction of Orthogonal Polynomials from a General Recurrence Formula.” Biometrics 24:695.

Fosse, Ethan and Christopher Winship. 2018. "Moore-Penrose Estimators of Age-Period-Cohort Effects: Their Interrelationship and Properties.” Sociological Science 5:304-334.

Fosse, Ethan and Christopher Winship. 2019a. "Analyzing Age-Period-Cohort Data: Critiques and New Directions.” Annual Review of Sociology 45:467-92.

Fosse, Ethan and Christopher Winship. 2019b. "Bounding Analyses of Age-Period-Cohort Effects." Demography.

Gaito, John. 1965. "Unequal Intervals and Unequal n in Trend Analyses.” Psychological Bulletin 63:125-127.

Holford, Theodore R. 1983. "The Estimation of Age, Period and Cohort Effects for Vital Rates.” Biometrics 39:311.

Luo, Liying, James Hodges, Christopher Winship, and Daniel Powers. 2016. "The Sensitivity of the Intrinsic Estimator to Coding Schemes: Comment on Yang, Schulhofer-Wohl, $\mathrm{Fu}$, and Land." American Journal of Sociology 122:930-961.

O’Brien, Robert M. 2011. “Constrained Estimators and Age-Period-Cohort Models.” Sociological Methods \& Research 40:419-452.

Rodgers, Willard L. 1982. “Estimable Functions of Age, Period, and Cohort Effects.” American Sociological Review 47:774. 